\title{
Neuromonitoraggio continuo in chirurgia tiroidea
}

\author{
Gianlorenzo Dionigi ${ }^{1}$. Pier Giorgio Calò ${ }^{2}$. Gabriele Materazzi ${ }^{3}$. Maurizio lacobone ${ }^{4}$. \\ Nadia Innaro ${ }^{5}$ Maurizio De Palma ${ }^{6}$. Luciano Pezzullo ${ }^{7}$. Maria Grazia Chiofalo ${ }^{7}$. \\ Alberto Zaccaroni ${ }^{8}$. Antonella Pino ${ }^{1}$. Paolo Carcoforo ${ }^{9}$. Annamaria D'Amore ${ }^{10}$. \\ Carmela De Crea ${ }^{10}$. Nicola Avenia ${ }^{11}$. Marco Raffaelli ${ }^{10}$. Giovanni Conzo ${ }^{12}$. Paolo Del Rio ${ }^{13}$. \\ Giovanni Docimo ${ }^{14}$. Mario Testini ${ }^{15} \cdot$ Celestino Pio Lombardi ${ }^{10}$
}

Accettato: 12 maggio 2020

(c) Springer Nature Switzerland AG

Sommario La tecnica del monitoraggio intraoperatorio continuo (CIONM) del nervo laringeo ricorrente (NLR) è riconosciuta come uno strumento utile per riconoscere le lesioni nervose imminenti e interrompere la relativa manovra chirurgica per prevenire le lesioni permanenti durante la tiroidectomia. Il CIONM fornisce costantemente preziose informazioni in tempo reale, il che è molto utile durante interventi chirurgici complessi della tiroide, specialmente in

Proposto da Gianlorenzo Dionigi.

Materiale elettronico supplementare La versione elettronica di questo articolo (https://doi.org/10.1007/s40619-020-00811-2) contiene materiale supplementare, disponibile per gli utenti autorizzati.

$凶$ G. Dionigi

gdionigi@unime.it

1 Divisione di Chirurgia Endocrina e Mininvasiva, Università degli Studi di Messina, Messina, Italia

2 Dipartimento di Chirurgia, Università di Cagliari, Monserrato, Cagliari, Italia

3 Divisione di Chirurgia, Università di Pisa, Pisa, Italia

4 Divisione di Endocrinochirurgia, Dipartimento di Chirurgia, Università di Padova, Padova, Italia

5 Divisione di Chirurgia, Università Magna Grecia, Catanzaro, Italia

6 Divisione di Chirurgia, Azienda Ospedaliera di Rilievo Nazionale A. Cardarelli, Napoli, Italia

7 Divisione Chirurgia Tiroide e Paratiroide, IRCCS G. Pascale, Napoli, Italia

8 Divisione Chirurgia Endocrina, AUSL Romagna-MorgagniPierantoni, Forlì, Italia

9 Dipartimento di Chirurgia, Università di Ferrara, Ferrara, Italia

10 Università Cattolica del Sacro Cuore, Roma, Italia

11 Divisione Chirurgia Endocrina, Università di Perugia, Perugia, Italia contesti di anatomia insolita. Il CIONM supera la limitazione metodologica inerente al neuromonitoraggio intermittente (IINOM). L'evento elettromiografico (EMG) combinato clinicamente importante (riduzione ampiezza e aumento latenza del segnale EMG), indicativo di imminente lesione del NLR, previene la maggior parte delle lesioni legate alla trazione del NLR anatomicamente intatto consentendo la modifica della manovra chirurgica che ne è causa nell' $80 \%$ dei casi. Come ulteriore estensione, il CIONM aiuta anche a identificare il recupero del nervo funzionale intraoperatorio, qualora avvenga una ripresa del valore di ampiezza $\geq 50 \%$ rispetto ai valori della baseline iniziale; ciò consente di continuare la resezione del lato controlaterale. Il CIONM facilita un'azione correttiva precoce prima che sia stato fatto un danno permanente al NLR. Il CIONM è una tecnica recente ma in rapida evoluzione, in via di perfezionamento da vari studi incentrati sul miglioramento della sua attuazione e interpretazione, nonché sull'eliminazione degli ostacoli tecnici.

Parole chiave Neuromonitoraggio intraoperatorio .

Neuromonitoraggio continuo - Tiroidectomia ·

Stimolazione del nervo vago $\cdot$ Paralisi del nervo laringeo ricorrente

12 Dipartimento di Scienze Mediche, Chirurgiche, Neurologiche, Metaboliche e dell'Invecchiamento, Università della Campania Luigi Vanvitelli, Caserta, Italia

13 Dipartimento di Chirurgia, Università di Parma, Parma, Italia

14 Dipartimento di Scienze Cardiotoraciche e Respiratorie, Università della Campania Luigi Vanvitelli, Caserta, Italia

15 Divisione Chirurgia Universitaria "V. Bonomo", Dipartimento di Scienze Biomediche e Oncologia Umana, Università di Bari, Bari, Italia 


\section{Introduzione}

L'identificazione e la dissezione del nervo laringeo ricorrente (NLR) di routine nella chirurgia endocrina del collo sono dimostrati come metodi sicuri e preziosi per proteggerlo dalle lesioni iatrogene [1]. La visualizzazione del NLR è considerata lo standard di riferimento per la prevenzione di lesioni del NLR durante la chirurgia tiroidea [2].

È noto che la principale fonte di disfunzione del NLR postoperatoria non è la sezione del nervo ma è una lesione funzionale, non strutturale, non visibile né all'occhio umano e/o durante chirurgia endoscopica [3]. Dall'introduzione del monitoraggio neurale intraoperatorio (IONM) nella chirurgia della tiroide, più di 50 anni fa, la tecnologia è notevolmente migliorata negli ultimi dieci anni $[4,5]$. L'uso della stimolazione intermittente del NLR e del nervo vago (NV) è ormai diventata una pratica adottata in numerosi centri specializzati in Italia. Il monitoraggio nervoso moderno, sia intermittente (monitoraggio nervoso intraoperatorio intermittente, IIONM), sia continuo (monitoraggio neurale intraoperatorio continuo, CIONM), può aiutare a identificare il NLR, a tracciare il suo corso durante la dissezione, diagnosticare la perdita di segnale (loss of signal, LOS) segmentale (tipo 1) e diffusa (tipo 2) e offrire, talvolta, la base per un eventuale cambiamento della strategia chirurgica in un intervento di tiroidectomia pianificata bilaterale (in caso di LOS al primo lato operato) (Fig. 1).

Il vantaggio principale del CIONM, che utilizza un elettrodo vagale impiantabile, temporaneo, rispetto al IIONM, è la sorveglianza in tempo reale della funzione del NLR durante la mobilizzazione della tiroide e la dissezione del NLR, che IIONM non può offrire [6]. Il sostanziale svantaggio dello strumento IIONM, che utilizza la sonda di stimolazione portatile, è conseguente alla sua limitazione che espone il NLR al rischio di lesioni tra due stimolazioni (Tabella 1). Pertanto, la compromissione funzionale del NLR può essere rilevata solo dopo che il danno si è già verificato. Pertanto il IIONM non offre l'opportunità di intraprendere le azioni chirurgiche critiche necessarie per evitare l'eventuale lesione del NLR [7].

Il CIONM, il sistema più recente di IONM, supera questa limitazione offrendo un monitoraggio del NLR in tempo reale, con la possibilità di un riconoscimento dell'imminente lesione del nervo e l'apprezzamento del recupero intraoperatorio della funzione nervosa dopo la riduzione e/o perdita del segnale elettromiografico (EMG) (Tabella 2, Fig. 2) [7, 8]. È importante notare che nell' acronimo CIONM il termine "continuo" si riferisce alla parola "monitoraggio", sottolineando la natura costante del monitoraggio durante l'intervento senza la necessità di interrompere l'operazione, una volta posizionato l'elettrodo vagale per la stimolazione nervosa. Il CIONM all'inizio può sembrare irragionevole e può
INTERMITTENTE
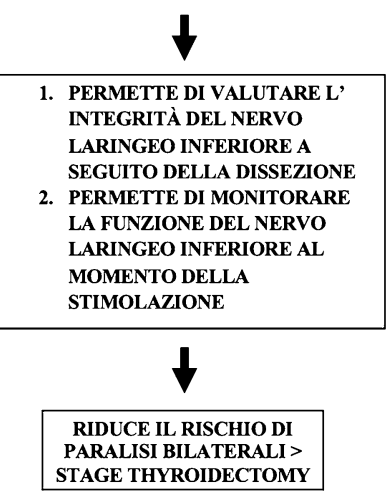

Fig. 1 Modalità di applicazione del neuromonitoraggio intraoperatorio. Il CIONM è stato introdotto come uno strumento superiore al monitoraggio intermittente. Il CIONM consente ai chirurghi di prendere misure intraoperatorie per invertire le manovre chirurgiche avverse per il NLR

essere visto come un'eccessiva stimolazione nervosa non fisiologica. È un noto principio di neurofisiologia che la stimolazione continua di un nervo motore periferico porta a una singola contrazione del muscolo innervato. Il CIONM può essere descritto più accuratamente come stimolazione pulsata ripetuta che è contemporanea alle manovre chirurgiche [9]. Il possibile vantaggio di un formato CIONM è che ha il potenziale per monitorare l'intero nervo laringeo ricorrente e, quindi, l'integrità funzionale del NLR in tempo reale durante l'intervento chirurgico e potrebbe identificare i segnali EMG associati a lesioni imminenti [8]. Come mostrato da Schneider e collaboratori [9], l'esito finale della disfunzione del NLR è stato significativamente migliorato dal CIONM.

\section{Sicurezza del neuromonitoraggio continuo}

Oltre a due segnalazioni aneddotiche di complicanze locali e cardiache durante CIONM in pochissimi pazienti, ci sono diversi dati clinici che non mostrano alcuna evidenza di avversità correlata alla dissezione circolare di un segmento del $\mathrm{NV}$ o intrinsecamente correlata a CIONM (Tabella 3) [10, $11]$.

È della massima importanza rendersi conto che la corrente da 1 a $2 \mathrm{~mA}$, utilizzata nella tecnica CIONM, non attiva le fibre $\mathrm{C}$ demielinizzate sottili responsabili dei maggiori effetti autonomici $[2,8]$. La stimolazione con $1 \mathrm{~mA}$ è sovramassimale solo per le fibre motrici A efferenti e le fibre B autonomiche mielinizzate. Questi risultati sono coerenti con i dati sugli animali, dimostrando che l'ampiezza del segnale EMG suscitato non può essere aumentata aumentando la corrente di stimolazione al di sopra di una soglia compresa tra 0,7 e $0,8 \mathrm{~mA}$ [9]. La stimolazione del nervo vago a frequenze inferiori a $30 \mathrm{~Hz}$ (la frequenza massima in CIONM 
L'Endocrinologo

Tabella 1 Nella chirurgia con il monitoraggio del NLR, il

\begin{tabular}{lll}
\hline Proprietà & IONM continuo & IONM intermittente \\
\hline Prevenire la paralisi transitoria del nervo laringeo inferiore & - & - \\
Prevenire la paralisi permanente del nervo laringeo inferiore & ++ & - \\
Sincronizzazione con le manovre chirurgiche & +++ & - \\
Contezza dello stress del nervo & +++ & + \\
Valutazione della ripresa funzionale del nervo & +++ & + \\
Tipo di lesione nervosa & ++ & + \\
Ricerca del nervo laringeo inferiore, mapping & + & ++ \\
Sito della lesione & + & ++ \\
Varianti anatomiche, ramificazione & + & ++ \\
Dissezione del nervo & - & ++ \\
Monitoraggio della branca esterna del nervo laringeo superiore & - & ++ \\
Malposizionamento del tubo endotracheale & ++ & - \\
Documentazione & +++ & + \\
\hline
\end{tabular}
IIONM e il CIONM sono complementari

Tabella 2 Al momento, sono disponibili vari tipi di elettrodi per il CIONM con diversi design e sono distinguibili in base all'estensione della dissezione richiesta per posizionarli sul NV

\begin{tabular}{|c|c|c|c|c|c|c|}
\hline & A "S" & Ancora & V3 & Delta & Sassofono & APS \\
\hline \multirow[t]{2}{*}{ Design } & & & & & & \\
\hline & Aperto & Aperto & Aperto & Imente chiuso & Parzialmente chiuso & Chiuso \\
\hline Dissezione del NV & Parziale & Parziale & & $<360^{\circ}$ & $360^{\circ}$ & $360^{\circ}$ \\
\hline Posizionamento & . & . & 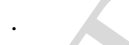 & $\cdot$ & . & . \\
\hline Misura (mm) & $54 \times 8 \times 0,8$ & $25 \times 0,8 \times 18$ & 20 & $18,05 \times 5 \times 7$ & $17,6 \times$ variabile & $2-3$ \\
\hline Adattabilità & . & . & & .. & .. & $\ldots$ \\
\hline Versatilità & . & . & & .. & .. & $\ldots$ \\
\hline Flessibilità & $\ldots$ & . & & .. & .. & $\ldots$ \\
\hline & . & . & & .. & .. & $\ldots$ \\
\hline Modalità di stimolazione & Tripolare & Bipolare & Tripolare & Bipolare & Tripolare & Monopolare \\
\hline Stabilità del segnale & . & .. & . & $\ldots$ & $\ldots$ & .... \\
\hline Compagnia & Langer & Langer & Inomed & Inomed & Langer & Medtronic \\
\hline Mercato CE & 2010 & 2009 & 2008 & 2013 & 2013 & 2011 \\
\hline Tipologia & Riutilizzabile & Riutilizzabile & - & Non riutilizzabile & Non riutilizzabile & Non riutilizzabile \\
\hline Ampiezza di impulso & $200 \mathrm{mcs}$ & $200 \mathrm{mcs}$ & $200 \mathrm{mcs}$ & $200 \mathrm{mcs}$ & $200 \mathrm{mcs}$ & $200 \mathrm{mcs}$ \\
\hline Intensità di stimolazione & $1-2,5$ & $1-2,5$ & $3-5$ & 1 & $1-2,5$ & 1 \\
\hline
\end{tabular}

è di $3 \mathrm{~Hz}$ ) non è stata associata a successivi effetti vagali avversi $[2,8]$. Inoltre, non si ritiene che la corrente di stimolazione da $1 \mathrm{~mA}$ utilizzata per il CIONM generi effetti concomitanti o conseguenti effetti vagali avversi che portano a sintomi centrali (mal di testa, intorpidimento), cardiaci (aritmie, bradicardia), polmonari (broncospasmo) o gastrointestinali (nausea, vomito) [9]. Gli studi che hanno applicato la corrente di stimolazione fino a 5,0 mA per il CIONM hanno osservato una maggiore variabilità della frequenza cardiaca, implicando uno squilibrio del sistema nervoso autonomo. L'attivazione parasimpatica evocata non era associata a un aumento compensativo dell' attività simpatica e rimaneva subclinica [9-15].

Se gli standard del IONM, inclusi la dissezione del NV e il RLN, vengono applicati in modo meticoloso, non vi sono prove che CIONM causi intrinsecamente danni. Inoltre, anche i pazienti più anziani con blocco AV avanzato e/o pacemaker possono essere monitorati in sicurezza da CIONM [12]. In letteratura sono stati anche riportati casi di applicazione sicura dell'elettrodo vagale nella chirurgia della tiroide robotica transascellare e di esofagectomia toracoscopica $[13,14]$. 
Fig. 2 Elettrodi per il CIONM Tutti i produttori (Medtronic, Jacksonville, USA; Inomed, Emmendingen, Germania; Dr. Langer Medical, Waldkirch, Germania) forniscono dispositivi di CIONM

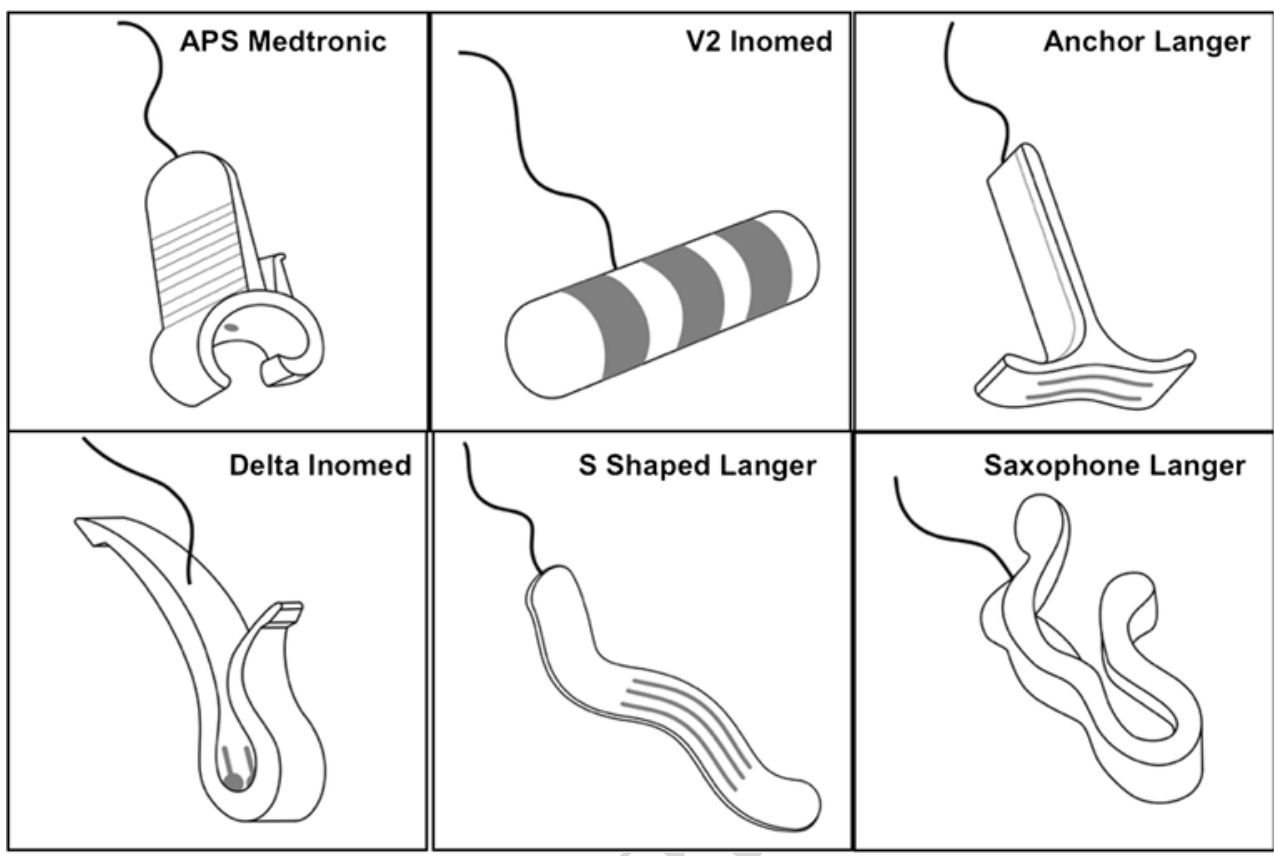

\section{Tecnica del CIONM}

La tecnica CIONM generalmente comprende un sistema con display EMG multicanale, il tubo endotracheale con elettrodi di superficie, elettrodo di stimolazione per il monitoraggio intermittente e l'elettrodo temporaneamente posizionato sul NV $[3,5,6]$.

Viene proposto l'uso sincronico sia della sonda di stimolazione IIONM per l'identificazione e del monitoraggio del corso del NLR, sia dell'elettrodo sul NV per il controllo in tempo reale della funzione del NLR (Tabella 1).

Per un uso sicuro e affidabile dell'elettrodo del CIONM dovrebbero essere considerati i punti successivi:

- accesso al NV all'interno della guaina carotidea prima di esporre la tiroide con approccio anteriore (linea mediana) o laterale (tra sternoioideo e muscolo sternocleidomastoideo) (Fig. 3);

- stimolazione del NV mediante sonda manuale di stimolazione per testare l'integrità e la funzione del nervo prima del posizionamento dell'elettrodo CIONM;

- dissezione circolare $\left(360^{\circ}\right)$ di un piccolo segmento di NV (a differenza del IIONM) (Fig. 3);

- stimolazione iniziale di $1 \mathrm{~mA}$;

- calibrazione (baseline) del sistema con la massima ampiezza ottenibile: si stabilisce che l'ampiezza della cosiddetta curva di riferimento baseline deve essere $\geq 500 \mu \mathrm{V}$ durante la calibrazione iniziale del sistema CIONM per garantire un segnale EMG stabile e affidabile [10]. I valori di ampiezza massima sono preziosi per due motivi: 1) l'ampiezza sufficiente è il prerequisito per il calcolo stabile e affidabile della latenza; e 2) maggiore è l'ampiez- za, maggiore è la tolleranza per le manovre correlate alla dissezione che preannunciano lesioni nervose imminenti; - una volta ottenuta la curva di riferimento (baseline) del CIONM, le modifiche (ampiezza e latenza) vengono visualizzate su una linea temporale. È possibile impostare valori di soglia, per aiutare il chirurgo a identificare manovre rischiose. Esporre la ghiandola tiroidea, l'identificazione di RLN e la sua dissezione sono identici a quelli di IIONM, usando la sonda di stimolazione portatile $[1,11$, $15]$.

\section{Riconoscimento delle lesioni nervose imminenti}

L'uso del CIONM richiede esperienza e osservazione del grafico EMG per rispondere rapidamente a segnali di allarme EMG [13].

La riduzione dell'ampiezza isolata inferiore al 50\% rispetto al valore basale o l'aumento della latenza isolata inferiore al $110 \%$ del basale può essere un artefatto, e si ritiene che derivi da malrotazione del tubo e/o dalla dislocazione tracheale con contatto compromesso tra gli elettrodi di superficie del tubo endotracheale e le corde vocali a seguito di manipolazione e trazione della tiroide [11]. Altre cause di scarsa registrazione e/o stimolazione durante CIONM sono: 1) perdita temporanea della registrazione EMG causata dalla coagulazione con bipolare; 2) la dislocazione degli elettrodi cutanei; 3) la dislocazione dell'elettrodo sul NV; e 4) una discrepanza tra le dimensioni del NV e l'elettrodo un allarme acustico e visivo quando vengono superati i 
L'Endocrinologo

Tabella 3 Revisione complicanze del CIONM riportate in letteratura

\begin{tabular}{|c|c|c|c|c|c|c|c|c|c|}
\hline $\begin{array}{l}\text { Autore } \\
\text { Rivista }\end{array}$ & Anno & $\begin{array}{l}\text { Probe } \\
\text { per } \\
\text { C-IONM }\end{array}$ & \# NV & $\begin{array}{l}\text { Intensità di } \\
\text { stimolazione } \\
\text { del NV }\end{array}$ & $\begin{array}{l}\text { Monitoraggio } \\
\text { eventi avversi }\end{array}$ & Eventi avversi & $\begin{array}{l}\text { Complicanze } \\
\text { locali }\end{array}$ & $\begin{array}{l}\text { Tempo } \\
\text { impiegato } \\
(\mathrm{min})\end{array}$ & $\begin{array}{l}\% \text { di malposi- } \\
\text { zionamento }\end{array}$ \\
\hline $\begin{array}{l}\text { Terris } \\
\text { WJS }\end{array}$ & 2015 & & 12 & $1 \mathrm{~mA}$ & $?$ & $\begin{array}{l}1 \text { (bradicardia, } \\
\text { ipotensione) }\end{array}$ & $\begin{array}{l}1 \text { lesione del } \\
\mathrm{NV}\end{array}$ & $\begin{array}{l}7,9 \pm 6,1 \\
(6-26)\end{array}$ & $41 \%$ \\
\hline $\begin{array}{l}\text { Schneider } \\
\text { LAS }\end{array}$ & 2009 & & 78 & $\begin{array}{l}1 \mathrm{~mA} \\
(0.8-2 \mathrm{~mA})\end{array}$ & $\begin{array}{l}\text { Anestesia } \\
\text { Monitor }\end{array}$ & 0 & 0 & $\begin{array}{l}1,45 \\
(0,56-5,25)\end{array}$ & $<5 \%$ \\
\hline $\begin{array}{l}\text { Mangano } \\
\mathrm{H} \& \mathrm{~N}\end{array}$ & 2015 & . & 400 & $1 \mathrm{~mA}$ & $\begin{array}{l}\text { Anestesia } \\
\text { Monitor }\end{array}$ & 0 & 0 & $15=>6$ & $11=>6 \%$ \\
\hline $\begin{array}{l}\text { Schneider } \\
\text { BJS }\end{array}$ & 2015 & & 2581 & $1 \mathrm{~mA}$ & $\begin{array}{l}\text { Anestesia } \\
\text { Monitor }\end{array}$ & 0 & & - & - \\
\hline $\begin{array}{l}\text { Phelan } \\
\text { Laryng. }\end{array}$ & 2014 & & 200 & $1 \mathrm{~mA}$ & $\begin{array}{l}\text { Anestesia } \\
\text { Monitor }\end{array}$ & 0 & 0 & 2,5 & - \\
\hline $\begin{array}{l}\text { Schneider } \\
\text { H\&N }\end{array}$ & 2013 & & 52 & $1 \mathrm{~mA}$ & $\begin{array}{l}\text { Anestesia } \\
\text { Monitor }\end{array}$ & 0 & 0 & 3,7 & $<5 \%$ \\
\hline $\begin{array}{l}\text { Jonas } \\
\text { STI }\end{array}$ & 2014 & & 1184 & $1-3 \mathrm{~mA}$ & $\begin{array}{l}\text { Anestesia } \\
\text { Monitor }\end{array}$ & 0 & 0 & 5,7 & 0 \\
\hline $\begin{array}{l}\text { Ulmer } \\
\text { Friedrich }\end{array}$ & 2012 & & 79 & $1 \mathrm{~mA}$ & $\begin{array}{l}\text { Variazioni } \\
\text { della FR }\end{array}$ & 0 & 0 & 6,8 & 0 \\
\hline $\begin{array}{l}\text { Van Slycke } \\
\text { LAS }\end{array}$ & 2013 & & 180 & $1 \mathrm{~mA}$ & $\begin{array}{l}\text { Anestesia } \\
\text { Monitor }\end{array}$ & 0 & 0 & 3,7 & 0 \\
\hline
\end{tabular}

Tabella 4 Definizione di perdita di segnale EMG (loss of signal, LOS). Definizione secondo le linee guida INMSG (Intraoperative Neural Monitoring Study Group)

\begin{tabular}{ll}
\hline- & Motilità cordale conservata alla laringoscopia preoperatoria \\
- & Segnale EMG iniziale soddisfacente $(\mathrm{V} 1>500 \mathrm{mcV})$ \\
- & Nessuna risposta EMG con una stimolazione a 1-2 mA \\
- & Bassi valori EMG con una stimolazione a 1-2 mA \\
- & Assenza di contrazione dei muscoli laringei \\
- & Applicazione dell'algoritmo per risoluzione dei problemi \\
\hline
\end{tabular}

CIONM può provocare scarse caratteristiche di stimolazione e/o indurre l'elettrodo a staccarsi.

Le variazioni del segnale EMG durante il CIONM sono classificate in: 1) variazioni non pericolose; 2) eventi EMG combinati; e 3) LOS in base al rischio relativo di lesioni del NLR (Tabelle 4, 5).

Per facilitare l'interpretazione di segnali EMG quantitativi clinicamente rilevanti durante il CIONM, sono stati definiti eventi EMG "combinati" avversi, che comprendono cambiamenti concordanti specifici sia nell'ampiezza che nella latenza del segnale EMG, definiti come una diminuzione maggiore del $50 \%$ in ampiezza e aumento maggiore del $110 \%$ della latenza rispetto ai valori basali. Questi eventi EMG combinati hanno dimostrano soglie EMG clinicamente importanti indicative di imminente lesione del NLR (Fig. 4).

Recentemente, è stato dimostrato in 1.314 nervi a rischio, in chirurgia tiroidea guidata da CIONM, che la reazione immediata ritirando la trazione nervosa non ha proceduto a LOS nell' $80 \%$ ( $n=63$ di 77) dei casi [16]. Questa correlazione elettrofisiologica della disfunzione nervosa imminente potrebbe verificarsi in due fasi di cambiamenti neuro-anatomici. La prima fase rappresenta la riduzione dell'ampiezza come riduzione del numero di neurofibre che trasmettono potenziale d'azione a causa della trazione sul NLR. La seconda fase con ulteriore riduzione dell'ampiezza e aumento della latenza potrebbe essere tradotta come un 
Tabella 5 Stratificazione del rischio in base ai cambiamenti del segnale EMG durante tiroidectomia con C-IONM (neuromonitoraggio intraoperatorio continuo) (baseline definita da ampiezza $>500 \mathrm{mcV}$ )

\begin{tabular}{|c|c|c|c|c|}
\hline \multirow[t]{2}{*}{ Definizione } & \multirow[t]{2}{*}{ Gruppo } & \multicolumn{2}{|l|}{ Parametri EMG } & \multirow[t]{2}{*}{ Rilevanza prognostica } \\
\hline & & Ampiezza & Latenza & \\
\hline \multirow{3}{*}{$\begin{array}{l}\text { Variazioni EMG non } \\
\text { pericolose }\end{array}$} & $1 \mathrm{a}$ & $\geq 50 \%$ & $\leq 110 \%$ & Assenza di paralisi cordale \\
\hline & $1 b$ & $\geq 50 \%$ & $>110 \%$ & Assenza di paralisi cordale \\
\hline & $1 \mathrm{c}$ & $<50 \% \mathrm{ma}>100 \mathrm{mcV}$ & $\leq 110 \%$ & Assenza di paralisi cordale \\
\hline Evento combinato (EC) & 2 & $<50 \% \mathrm{ma}>100 \mathrm{mcV}$ & $>110 \%$ & $\begin{array}{l}\text { Assenza di paralisi cordale quando EC non è } \\
\text { seguito da perdita di segnale }\end{array}$ \\
\hline \multirow[t]{3}{*}{ Perdita di segnale } & $\begin{array}{l}\text { 3a (lesione } \\
\text { segmentaria; } \\
\text { tipo 1) }\end{array}$ & $\leq 100 \mathrm{mcV}$ & Qualsiasi & $\begin{array}{l}\text { Senza ripresa o con una ripresa di ampiezza } \\
\text { intraoperatoria }<50 \% \text { della baseline: paralisi } \\
\text { cordale }\end{array}$ \\
\hline & & & & $\begin{array}{l}\text { Con ripresa di ampiezza intraoperatoria } \geq 50 \% \text { della } \\
\text { baseline: assenza di paralisi cordale }\end{array}$ \\
\hline & $\begin{array}{l}3 \text { b (lesione diffusa; } \\
\text { tipo 2) }\end{array}$ & $\leq 100 \mathrm{mcV}$ & Qualsiasi & $\begin{array}{l}\text { Senza ripresa o con una ripresa di ampiezza } \\
\text { intraoperatoria }<50 \% \text { della baseline: } 70 \% \text { paralisi } \\
\text { cordale }\end{array}$ \\
\hline
\end{tabular}

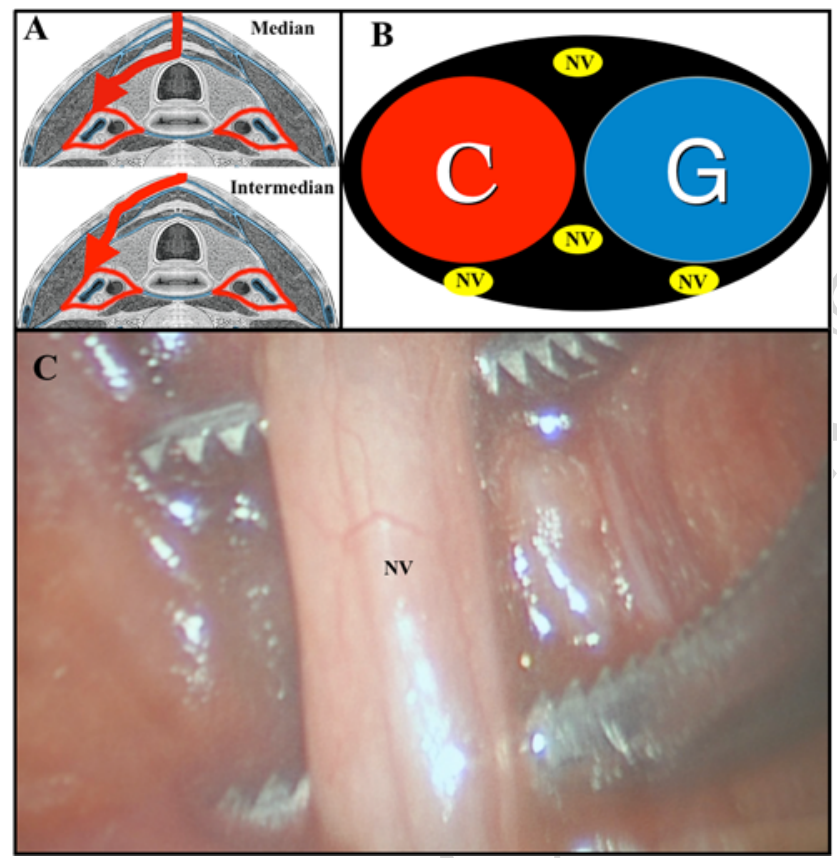

Fig. 3 Posizionamento dell'elettrodo per il CIONM. a Diversi accessi al NV. Il vantaggio dell'incisione sulla linea mediana è l'uso di piccole incisioni cutanee, come per la chirurgia benigna della tiroide. L'approccio più laterale alternativo impiegato soprattutto nell'enorme gozzo della tiroide, nelle recidive e nella chirurgia del carcinoma tiroideo presenta il vantaggio di un tasso inferiore di dislocazione accidentale dell'elettrodo CIONM poiché il filo elettrico è al di fuori del campo operativo; b differenti sedi del NV rispetto alla carotide $(C)$ e giugulare $(G)$; c dissezione a $360^{\circ}$ del NV necessaria per il posizionamento dell'elettrodo CIONM. È di fondamentale importanza evitare la devascolarizzazione del NV durante il suo isolamento e mobilizzazione

ulteriore aumento dell'intervallo di tempo all'azione del muscolo della corda vocale mediante una continua trazione del
NLR mentre il numero di miofibre è già stato ridotto. La nostra comprensione di queste possibili fasi è ancora nascente poiché il trauma del NLR in questi casi è speculativo e non quantificato.

L'esperienza con il CIONM ha dimostrato che proprio all'inizio di un evento combinato EMG, la trazione sul NLR dovrebbe essere immediatamente rilasciata riposizionando la tiroide e quindi bisogna attendere che l'ampiezza aumenti di nuovo a oltre il 50\% dell' ampiezza di base. Se si verifica più volte una riduzione ripetuta dell' ampiezza correlata alla trazione al di sotto del $50 \%$ della linea di base, l'approccio chirurgico al lobo tiroideo deve essere modificato con diversa dissezione per prevenire ulteriori e pericolosi cambiamenti EMG. Al contrario, se l'evento combinato permane, come pure l'approccio chirurgico, si può avanzare a LOS (perdita di segnale) (che in genere è significativamente meno reversibile) quindi a paralisi cordale postoperatoria [1].

\section{Riconoscimento del recupero della funzione nervosa dopo LOS}

Le lesioni "segmentali" (tipo 1) del NLR sono causate da un trauma diretto al nervo, come la sezione del nervo, legatura, o coagulazione bipolare. Le lesioni nervose diffuse (tipo 2) potrebbero essere il risultato di forze globali più indirette, in seguito a una maggiore trazione globale sulle vie aeree o sui tessuti adiacenti al nervo [6]. Le lesioni segmentarie e diffuse del nervo differiscono notevolmente in termini di ripristino della perdita di segnale (mediane di 2 contro 156 secondi; $p$ log-rank <0,001) [16]. A differenza della maggior parte dei traumi meccanici, delle lesioni termiche o da 

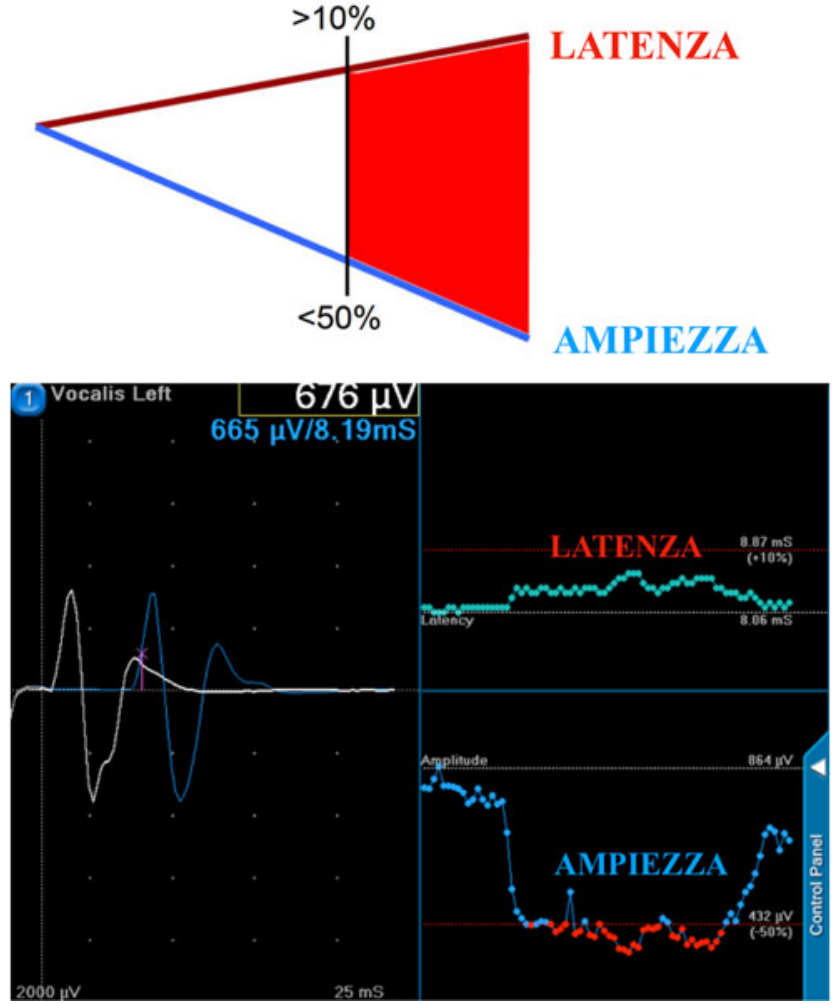

Fig. 4 NLR con imminente lesione: evento EMG combinato di riduzione di ampiezza e aumento di latenza. Gli eventi EMG "combinati" avversi, comprendono cambiamenti concordanti specifici sia nell'ampiezza che nella latenza del segnale EMG, definiti come una diminuzione maggiore del $50 \%$ in ampiezza e aumento maggiore del $110 \%$ della latenza rispetto ai valori basali. È possibile impostare un allarme acustico e visivo quando vengono superati i valori di soglia, per aiutare il chirurgo a identificare manovre rischiose

sezione in cui il segnale di monitoraggio del nervo è stato perso all'improvviso, le lesioni da trazione del NLR sono sempre state precedute da eventi EMG combinati (aumento latenza + riduzione ampiezza) (Fig. 4). Poiché la LOS segmentale ha meno probabilità di provocare cambiamenti EMG parziali graduali che possono essere identificati prima che si sia verificata una lesione definitiva, il CIONM potrebbe non aiutare a prevenire questo tipo di lesione. Ancora una volta l'insorgenza di cambiamenti avversi EMG nel tipo 1 o nel tipo 2 è probabilmente correlata al tipo, all'entità e al decorso del trauma ad esso associato (Figg. 5, 6).

L'esperienza del chirurgo e una meticolosa microdissezione sono fondamentali per ridurre al minimo queste lesioni, dal momento che alcune impostazioni anatomiche come nervi sottili, ramificazione extralaringea e nervi che corrono anteriormente all' arteria tiroidea inferiore aumentano il rischio di lesioni segmentali del nervo [10].

$\mathrm{Al}$ contrario, il CIONM può prevenire lesioni da trazione poiché la trazione può provocare eventi combinati EMG (aumento latenza + riduzione ampiezza), condizionando la modifica della manovra chirurgica sottostante [11].

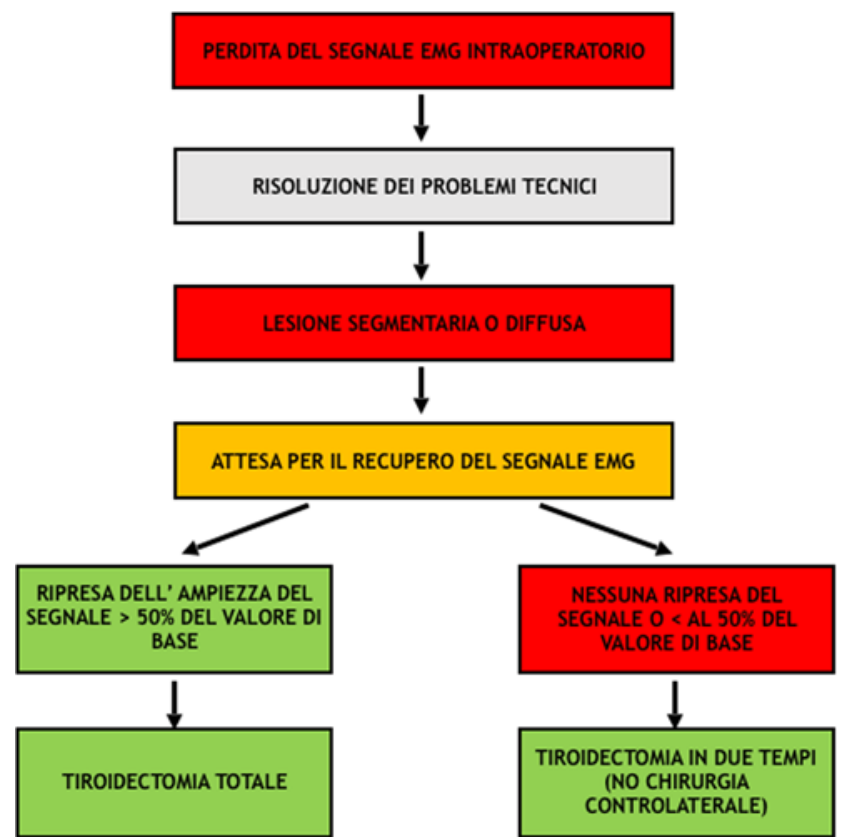

Fig. 5 Cambiamento intraoperatorio della strategia chirurgica con ausilio del CIONM

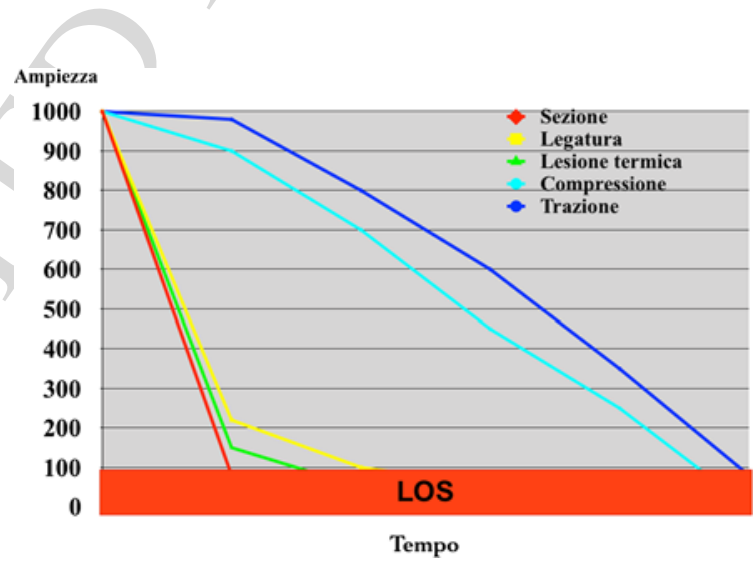

Fig. 6 Differenti profili di riduzione del segnale EMG a seconda del tipo di lesione del NLR

L'esperienza e la recente letteratura descrivono due diversi tipi di recupero intraoperatorio:

1. recupero incompleto (coerente con la paralisi cordale postoperatoria): l'ampiezza aumenta $>100 \mu \mathrm{V}$ ma $<50 \%$ del basale [9];

2. ripristino completo (coerente con la funzione delle corde vocali intatte nel postoperatorio): aumento dell' ampiezza $a \geq 50 \%$ della linea di base [9].

L'analisi di nervi a rischio con LOS e recupero EMG intraoperatorio ha mostrato una correlazione del grado di recupero dell' ampiezza intraoperatoria (\% del basale) dopo LOS con paralisi cordale postoperatoria. Più bassa è la percentuale di recupero dell'ampiezza (30 vs 40 vs 50\%) rispetto ai 
valori di base, maggiore è la percentuale di paralisi cordale postoperatoria (36 vs 22 vs 0\%) (dati non pubblicati).

Come mostrato di recente in letteratura, la funzione nervosa si è completamente ripristinata dopo LOS entro i primi 20 minuti (lesione NLR segmentale: 6,9 min; lesione NLR diffusa: $15,6 \mathrm{~min}$ ), il che significa che il processo decisionale dovrebbe essere fatto dopo 20 minuti di tempo di attesa [9]. Se l'ampiezza non ha recuperato o ripristina solo $<50 \%$ del basale, non è consigliabile attendere più di 20 minuti, poiché è noto che tutti $(n=16 / 16)$ i pazienti con lesione del nervo segmentale o tre quarti $(n=13 / 18)$ dei pazienti con lesione diffusa presentavano una funzionalità ridotta delle corde vocali. È evidente che la gravità della disfunzione delle corde vocali era correlata alla gravità della disfunzione nervosa. Tre quinti $(n=10 / 16)$ di pazienti con grave lesione segmentaria del nervo senza o con recupero EMG incompleto hanno mostrato paralisi cordale completa, mentre solo un terzo $(n=4 / 13)$ dei pazienti con lesione del NLR diffusa ha mostrato disfunzione completa delle corde vocali, indicando danni meno gravi.

\section{Cambiamento intraoperatorio della strategia chirurgica}

Si raccomanda una stretta aderenza all'approccio $L 1$ (laringoscopia preoperatoria), $V 1$ (stimolazione del NV prima della dissezione tiroidea), $R 1$ (stimolazione del NLR alla sua identificazione), $R 2$ (stimolazione del NLR al termine della dissezione), $V 2$ (stimolazione del $\mathrm{NV}$ al termine della dissezione), $L 2$ (laringoscopia postoperatoria) che è destinata a migliorare l'affidabilità del neuromonitoraggio [17].

Il CIONM ha un'affidabilità superiore al IIONM, con sensibilità del $90 \%$, specificità $99 \%$ e valore predittivo positivo dell' $88 \%$ [13]. L'accuratezza predittiva del CIONM è molto alta $(99,5 \%)$ e rappresenta una base per il processo decisionale intraoperatorio a favore o contro la chirurgia controlaterale. Le percentuali più basse di falsi positivi e falsi negativi con CIONM rispetto a IIONM $(0,3$ vs, $0,5 \% ; 0,2$ vs $0,6 \%$; ns) possono ridurre ulteriormente il numero di procedure temporanee non necessarie in falsi positivi e di potenziale paralisi cordale bilaterale in risultati falsi negativi, in particolare quando il concetto di recupero dell' ampiezza incompleta e completa dopo la LOS è incorporato nel processo decisionale nella tiroidectomia guidata dal CIONM $[13,16]$.

Pertanto, il CIONM offre la possibilità, se necessario, di modificare la strategia chirurgica dopo LOS (previa valutazione oculata dell'algoritmo di risoluzione dei problemi per LOS) (Fig. 5).

Una volta confermata la LOS, un periodo di attesa di 20 minuti consentirà al chirurgo di sapere se il nervo interessato si riprenderà completamente o meno, e se la chirurgia della tiroide controlaterale deve essere presa in considerazione dopo il completamento della prima resezione. Infatti, un nervo lesionato, indipendente dal tipo di LOS, richiede un'attesa minima di 20 minuti per recuperare $\geq 50 \%$ della sua ampiezza di base prima di procedere con il completamento della tiroidectomia controlateralmente [9]. Se l'ampiezza del nervo non riesce a recuperare almeno il 50\% della sua ampiezza di base dopo 20 minuti, esiste un rischio dell' $85 \%$ ( $n=29 / 34$ pazienti) di una paralisi della corda vocale nel postoperatorio [9]. Questi dati aiutano il chirurgo a riconsiderare eventualmente il piano chirurgico, in particolare nel contesto della LOS sul primo lato della resezione in un intervento chirurgico programmato bilaterale.

Il completamento della tiroidectomia, dopo il recupero della funzione della corda vocale, dovrebbe essere disegnato come approccio logico dopo il recupero della funzione della corda vocale [18]. Come mostrato in letteratura, le lesioni del NLR "diffuse" hanno avuto un tempo di recupero più rapido della funzione delle corde vocali rispetto alle lesioni "segmentali" (mediane di 27 contro 62 giorni; $p<0,01$ ) [9, 16]. In assenza di un completo recupero intraoperatorio dell'ampiezza, la chirurgia controlaterale deve essere eseguita dopo attenta rivalutazione, con il consenso ulteriore del paziente.

\section{Limiti del CIONM}

Il CIONM ha alcuni limiti, sia tecnici che interpretativi del segnale EMG [19].

\section{Semplificazione}

La fase di posizionamento chirurgico dell'elettrodo CIONM deve essere semplificata in quanto richiede tempo ed è considerata più complessa rispetto al monitoraggio intermittente (che non richiede la dissezione del NV). Nuovi approcci al NV sono stati proposti per ottenere una procedura più semplice. I primi test con prototipi di modelli CIONM transcutanei o percutanei hanno dimostrato una fattibilità significativa [2]. Tali nuove alternative per CIONM possono migliorare la sicurezza e la semplificazione.

\section{Curva di apprendimento}

Certamente, nonostante i noti benefici del CIONM, l'adeguata formazione rimane il gold standard per una procedura CIONM sicura. Attualmente, non ci sono dati disponibili sulla curva di apprendimento CIONM, sia inerenti alla componente tecnica (ovvero, utilizzo e impostazione corretta dell'attrezzatura CIONM) sia alla componente interpretativa (è la persona che esegue il monitoraggio continuo in grado di distinguere tra una vera risposta EMG rispetto a un artefatto di stimolazione?). 


\section{Soglia allarmi}

Un avviso di allarme EMG durante CIONM deve essere generato da: 1) perdita di ampiezza superiore al $50 \%$; 2) aumento della latenza superiore al $10 \%$; 3) numero di registrazioni di eventi combinati; 4) eventi sincroni e logicamente associati alla manovra chirurgica a rischio; e 5) fattori sistemici, anestetici, tecnici devono essere esclusi. La severità dei criteri per generare un allarme è un fattore importante che è responsabile dei diversi tassi di falsi positivi e falsi negativi. L'uniformità nei criteri per gli avvisi è essenziale. Se viene utilizzata una soglia bassa per generare un allarme, allora vi è una maggiore possibilità di falsi positivi, con procedure chirurgiche che vengono inutilmente modificate $o$ abbandonate. Al contrario, se viene utilizzata una soglia elevata per l'innalzamento di un allarme, la possibilità di falsi negativi con conseguenti deficit neurologici postoperatori è un problema. Pertanto, sono sicuramente necessarie ulteriori ricerche dal settore clinico per definire in modo più completo i parametri per determinare corrette e giustificate soglie di allarme EMG durante il CIONM.

\section{La paralisi del NLR si verifica anche con CIONM}

Ancora più importante, il chirurgo deve sapere che anche con il CIONM può verificarsi la paralisi del NLR. Il CIONM consente di riconoscere e modificare l'azione chirurgica in caso di stress dilazionato nel tempo/costante, non acuto, del NLR (ad esempio uno stress da trazione, o compressione). Invece, in caso di danni rapidi, acuti e improvvisi (ad esempio lesioni termiche, o sezione del nervo) il chirurgo non può modificare la sua manovra chirurgica. Il CIONM consente di modificare l'azione chirurgica per alcuni profili EMG (non acuti) di variazione combinata dell'ampiezza e della latenza (Fig. 6).

\section{Conclusioni}

La tecnica CIONM è un possibile adiuvante per il chirurgo durante la chirurgia di tiroide, fornendo uno strumento utile per prevenire manovre dannose.

In casi particolarmente complessi come patologie recidive con numerose aderenze, tumori che si infiltrano nelle strutture adiacenti, enormi gozzi con invasione mediastinica o deviazione tracheale, il monitoraggio continuo è uno strumento da considerare utile ad assistere il chirurgo durante le rischiose e complesse procedure. In queste circostanze, l'identificazione precoce del RLN e del suo percorso non è sempre possibile; nonostante ciò, una volta posizionato l'elettrodo vagale del CIONM, l'EMG ottenuto durante il CIONM può avvisare il team costantemente e in tempo reale sulla sicurezza di ogni passaggio chirurgico.
L'evento combinato di riduzione di ampiezza di segnale e aumento della latenza è un segno elettromiografico patognomonico di imminente disfunzione nervosa che può progredire verso la LOS, per la quale il CIONM è attualmente il miglior sistema di allarme disponibile che richiede un'immediata modifica della manovra chirurgica e, quindi, della trazione nervosa. Al contrario, la lesione diretta che porta alla lesione segmentaria della NLR con improvvisa riduzione dell'ampiezza può ancora essere evitata solo con una dissezione nervosa meticolosa e non con il IONM.

Infine, la frequenza delle paralisi del NLR permanente è più bassa con CIONM e l'accuratezza nella previsione della motilità postoperatoria (conservata o meno) delle corde vocali è superiore al IIONM.

Conflitto di interesse Gli autori Gianlorenzo Dionigi, Pier Giorgio Calò, Gabriele Materazzi, Maurizio Iacobone, Nadia Innaro, Maurizio De Palma, Luciano Pezzullo. Maria Grazia Chiofalo, Alberto Zaccaroni, Antonella Pino, Paolo Carcoforo, Annamaria D'Amore, Carmela De Crea, Nicola Avenia, Marco Raffaelli, Giovanni Conzo, Paolo Del Rio, Giovanni Docimo, Mario Testini e Celestino Pio Lombardi dichiarano di non avere conflitti di interesse.

Consenso informato Lo studio presentato in questo articolo non ha richiesto sperimentazione umana.

Studi sugli animali Gli autori di questo articolo non hanno eseguito studi sugli animali.

Nota della casa editrice Springer Nature rimane neutrale in riguardo alle rivendicazioni giurisdizionali nelle mappe pubblicate e nelle affiliazioni istituzionali.

\section{Bibliografia}

1. Zhao Y, Li C, Zhang D et al (2020) Continuous neural monitoring in endoscopic thyroidectomy: feasibility experimental study for transcutaneous vagal nerve stimulation. J Laparoendosc Adv Surg Tech A 30(10):1095-1101

2. Zhang D, Wang T, Zhao Y et al (2020) Pre-prototype stimulating and recording endotracheal tube for continuous monitoring of the recurrent laryngeal nerve during thyroid surgery. J Invest Surg 9:1-11

3. Zhang D, Pino A, Caruso E et al (2020) Neural monitoring in thyroid surgery is here to stay. Gland Surg 9(Suppl 1):S43-S46

4. Sun H, Carcoforo P, Dionigi G (2020) Prerequisites for introducing neural monitoring in thyroid surgery. Eur Ann Otorhinolaryngol Head Neck Dis 137(1):91

5. Sun H, Kim HY, Dionigi G (2020) Neural monitoring is not a substitute to laryngeal examination in thyroid surgery. Surgery 167(5):883-884

6. Sun H, Kim HY, Carcoforo P, Dionigi G (2019) Cost and training are diffusion patterns limits for neural monitoring in thyroid surgery. Gland Surg 8(4):334-335

7. Zhang D, Li S, Dionigi G et al (2020) Stimulating and dissecting instrument for transoral endoscopic thyroidectomy: proof of concept investigation. Surg Endosc 34(2):996-1005

8. Wu CW, Huang TY, Chen HC et al (2019) Intra-operative neural monitoring of thyroid surgery in a porcine model. J Vis Exp. https://doi.org/10.3791/57919 
9. Schneider R, Randolph GW, Sekulla C et al (2013) Continuous intraoperative vagus nerve stimulation for identification of imminent recurrent laryngeal nerve injury. Head Neck 35:1591-1598

10. Terris DJ, Chaung K, Duke WS (2016) Mounting evidence of the potential perils associated with continuous intraoperative neuromonitoring: reply. World J Surg 40:770-771

11. Brauckhoff K, Vik R, Sandvik L et al (2016) Impact of EMG changes in continuous vagal nerve monitoring in high-risk endocrine neck surgery. World J Surg 40:672-680

12. Schneider R, Machens A, Bucher M et al (2016) Continuous intraoperative monitoring of vagus and recurrent laryngeal nerve function in patients with advanced atrioventricular block. Langenbeck's Arch Surg 401:551-556

13. Lörincz BB, Möckelmann N, Busch CJ et al (2016) Automatic periodic stimulation of the vagus nerve during single-incision transaxillary robotic thyroidectomy: feasibility, safety, and first cases. Head Neck 38:482-485

14. Tsang RK, Law S (2016) Adaptation of continuous intraoperative vagus nerve stimulation for monitoring of recurrent laryngeal nerve during minimally invasive esophagectomy. World J Surg 40:137-141
15. Liddy W, Lawson BR, Barber SR et al (2018) Anterior laryngeal electrodes for recurrent laryngeal nerve monitoring during thyroid and parathyroid surgery: new expanded options for neural monitoring. Laryngoscope 128(12):2910-2915

16. Schneider R, Sekulla C, Machens A et al (2015) Postoperative vocal fold palsy in patients undergoing thyroid surgery with continuous or intermittent nerve monitoring. Br J Surg 102:1380-1387

17. Randolph GW, Dralle H et al (International Intraoperative Monitoring Study Group) (2011) Electrophysiologic recurrent laryngeal nerve monitoring during thyroid and parathyroid surgery: international standards guideline statement. Laryngoscope 121(Suppl 1):S1-S16

18. Wu CW, Dionigi G, Barczynski M et al (2018) International neuromonitoring study group guidelines 2018: Part II: Optimal recurrent laryngeal nerve management for invasive thyroid cancerincorporation of surgical, laryngeal, and neural electrophysiologic data. Laryngoscope 128(Suppl 3):S18-S27

19. Sun H, Wu CW, Catalfamo A et al (2019) Limits of continuous neural monitoring in thyroid surgery. Updates Surg 71(1):187-188 